\title{
Angular Temperature Variations in a Wall-Cooled Packed-Bed Reactor
}

\author{
E. P. S. Schouten and K. R. Westerterp \\ Chemical Reaction Engineering Laboratories, Dept. of Chemical Engineering, University of Twente, \\ 7500 AE Enschede, The Netherlands
}

\begin{abstract}
The significance of the statistical character of wall-cooled packed-bed reactors was studied by measuring angular temperature variations, which result from the random nature of the packing. These are neglected in present-day mathematical models designed to describe the reactor behavior. The amplitude of these variations was determined experimentally as a function of the mass flux and the position in the tube, under conditions of heat transfer as well as of reaction. Angular variations are significant whenever radial temperature gradients are large.

Different methods used to average observed variations were surveyed. For the system presented, the angular variations have only a limited influence on the reaction rates if the angular averaged temperature is used. Thus, a two-dimensional deterministic continuum model can be used, notwithstanding the statistical character of the packed bed.
\end{abstract}

\section{Introduction}

Accurate mathematical modeling of wall-cooled packedbed reactors is desirable for a reliable design procedure. $\mathrm{Re}$ gretfully, the results of applying present-day models are unsatisfactory (see, e.g., Hoffman, 1979; Vortmeyer and Haidegger, 1991; Schouten et al., 1994). Almost exclusively models have been used that are referred to as deterministic continuum models because they contain parameters that are determined as constants over the entire bed and consider the gas and the solid phase, respectively, as a continuum. However, the actual structure of the bed is random: on a local scale the packing arrangement and hence also the velocity, the temperature, and the heat transport to a certain extent will be distributed in space. A continuum approach with fixed effective parameters over the entire bed may not be appropriate to describe this system accurately. Therefore, some authors have suggested that the statistical character of a packed bed should be incorporated in the reactor model (see $\mathrm{Fe}$ doseev and Shanin, 1986; Wijngaarden and Westerterp, 1992a,b; Borkink et al., 1992).

The random nature of the packing is observed if temperature measurements are made around a circular circumference. Variations in temperature in the angular direction have

Correspondence concerning this article should be addressed to K. R. Westerterp. been observed to be significant compared to radial and axial temperature differences in the packed bed. Dixon (1993) has made an extensive study of angular temperature variations in stationary heat-transfer experiments. The temperatures were measured above the packing. Borman and Westerterp (1992) have measured in the bed itself and found variations in the radial temperature profile after repacking that they explained by angularly varying temperatures. They briefly discuss the implications of such variations. A systematic study of angular temperature variations in a packed bed with reaction has not yet been made. Also some questions have not been answered yet: Do angular temperature variations depend on reaction? Are these related to axial and radial temperature differences? Does the mass flux have an influence? Can the neglect of angular variations explain the discrepancies between experimental results and model calculations with deterministic continuum models?

In this article, we try to answer these questions. Stationary temperature measurements have been conducted in a cylindrical packed bed, both under nonreacting as well as reacting conditions. The amplitude of the angular temperature variations will be determined as a function of position and of mass flux. We also will determine whether these variations are significant for the application of a continuum deterministic model. 


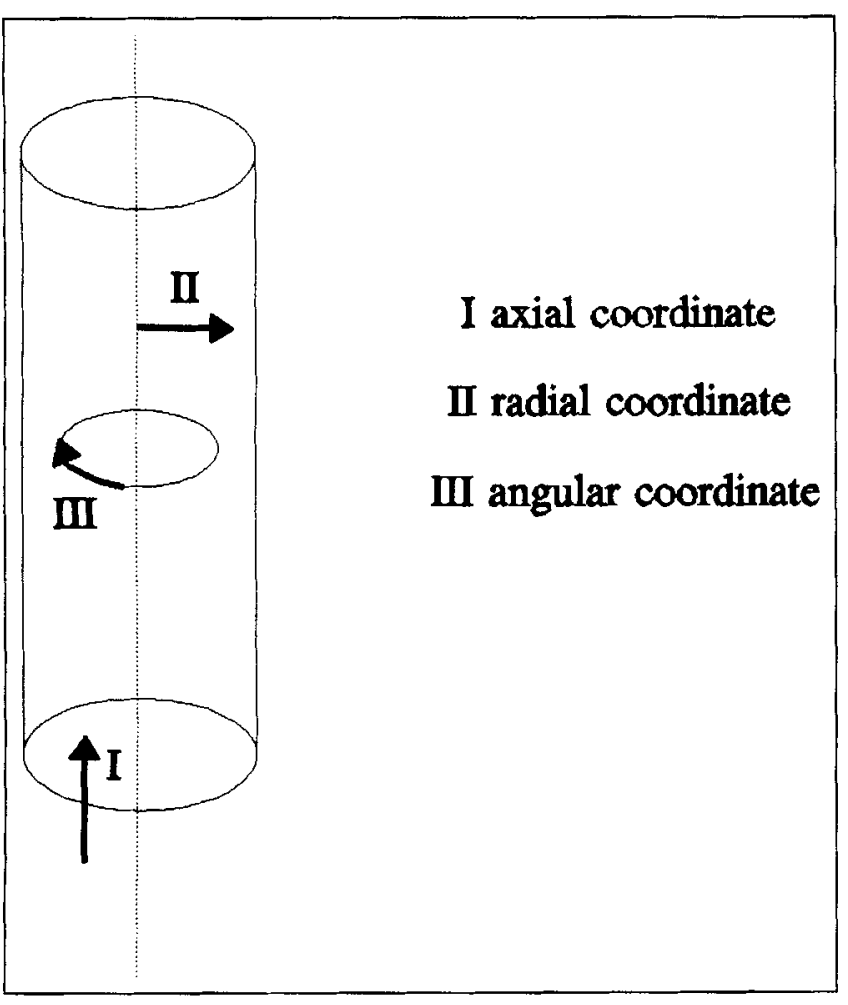

Figure 1. Three coordinates in a tubular reactor.

\section{Theoretical Backgrounds Angular variations}

Three directions can be distinguished in cylindrical reactors (see Figure 1): an axial coordinate in the direction of convective flow, a radial coordinate perpendicular to the axial coordinate being the direction for heat transport to or from the wall, and an angular coordinate. Models take a varying number of coordinates into account. A one-dimensional model only contains an axial coordinate, and heat transport to the wall is taken care of with an overall heattransfer coefficient to the wall. A so-called mean-cup temperature $T_{M C}$ is introduced as the average of the temperatures over a radial slice:

$$
T_{M C}=\frac{2}{R_{t}^{2}} \int_{r=0}^{R_{t}} r \cdot T[r] \cdot d r
$$

In a two-dimensional model both the axial as well as the radial direction are taken into account. Nearly all the literature to date has focused on temperature gradients in these two directions. Temperature variations in the angular coordinate are considered to be secondary in importance, and consequently have been ignored. Angular variations in packed beds do exist (see, e.g., Cresswell, 1986; Borman and Westerterp, 1992; Dixon, 1993). Deviations in temperature have been observed of up to to $12^{\circ} \mathrm{C}$ around the average temperature for experiments with a total temperature difference over the radius of about $40^{\circ} \mathrm{C}$. The reported experimental results apply to heat transfer only.

All authors have observed angular variations increases in the neighborhood of the wall (see, e.g., Dixon and Paterson,
1978; Cresswell, 1986; Giudici and do Nascimento, 1994). Thus the amplitude apparently depends on the radial position in the tube. The angular spread also has been found to be larger for shorter bed lengths (see, e.g., Dixon, 1993; Giudici and do Nascimento, 1994). According to Dixon (1993), the reduced amplitude of the angular variations in temperature merely reflects the approach of the thermal field to a uniform temperature. It was also argued that over longer bed lengths the gas has undergone an axial averaging by contacts with so many particles, consequently differences caused by variation of streamlines through the packing should average out, unless there is a bypass in some part of the bed (see also Borkink et al., 1992).

The flow rate was found to not influence the position of angular deviations (see Dixon, 1993), so the distribution of angular variations is specific for a certain packing arrangement: only by repacking the bed the distribution of angular variations will change. No quantification is given of the effect of flow rate on the amplitude of the angular temperature variations. Dixon (1993) has shown that, as $d_{t} / d_{p}$ decreases from 14 to 1.4 , angular variations become so large that radial profiles lose symmetry. Thus, for a smaller number of particles on a diameter the statistical character of the bed becomes more important. No regular periodic temperature variations were observed for any $d_{t} / d_{p}$ value, so Dixon (1993) concluded the variations are apparently random.

\section{Angular averaging}

Irregular axial and radial temperature profiles are found in practice, which are caused by the random nature of the packed bed and the discrete nature of the temperature measurements. Fitting such profiles to the smooth profiles obtained by continuum modeling may result in bad estimates for the effective transport coefficients (see Dixon, 1988). For a wall-cooled packed-bed reactor deviations from a smooth profile are more important in the radial direction compared to the axial direction in view of larger temperature gradients. In recent literature it therefore has been suggested that angular variations be evened out to obtain "suitable" radial profiles. Three different methods are discussed here.

One approach is to locate temperature sensors at several angular positions and average them around a circular circumference:

$$
\langle T\rangle=\frac{1}{2 \pi} \int_{\theta=0}^{2 \pi} T[\theta] \cdot d \theta .
$$

It has been shown that smooth radial profiles are obtained with this method if sufficient temperatures are measured even at small ratios of tube-to-particle diameter. At least eight thermocouples are necessary to this end, according to Dixon (1993). The variation observed around $\langle T\rangle$ is treated as though it was due to a random measurement error that contributes to the standard deviation of obtained parameters. Dixon (1988) reports an error in the obtained radial Bodenstein number of about $10 \%$ and an error in the Biot number at the wall of about $20 \%$, which he mainly attributes to angular temperature variations.

Another approach to average angular variations is repacking the bed (see Borman and Westerterp, 1992; Borkink et 
al., 1992; and Wijngaarden and Westerterp, 1992b). Angular variations are specific for certain packing and repacking results in a different radial profile for the same operating conditions. Smooth radial profiles can also be obtained by averaging these different profiles; Borkink and Westerterp (1994) repacked a bed 5 to 7 times, Wijngaarden and Westerterp (1992b) 20 times. Although repacking changes the local bed structure, and thus the local rate of heat transport, almost no differences have been found in bed scale parameters like the effective radial conductivity (see Borkink et al., 1992), and the conversion (see Borman and Westerterp, 1992). Dixon (1994) has shown for a wide range of packings that angular variations and repacking variations are statistically the same for a wide range of packings.

A third method was introduced by Giudici and do Nascimento (1994), who conducted heat-transfer experiments with air for six different packing materials. They developed a ring-shaped temperature sensor consisting of four copper wires with a thermocouple soldered in each of them (see Figure 2). Temperature measurements were performed above the packing. It was proved both experimentally as well as theoretically that the mean angular temperature was measured by

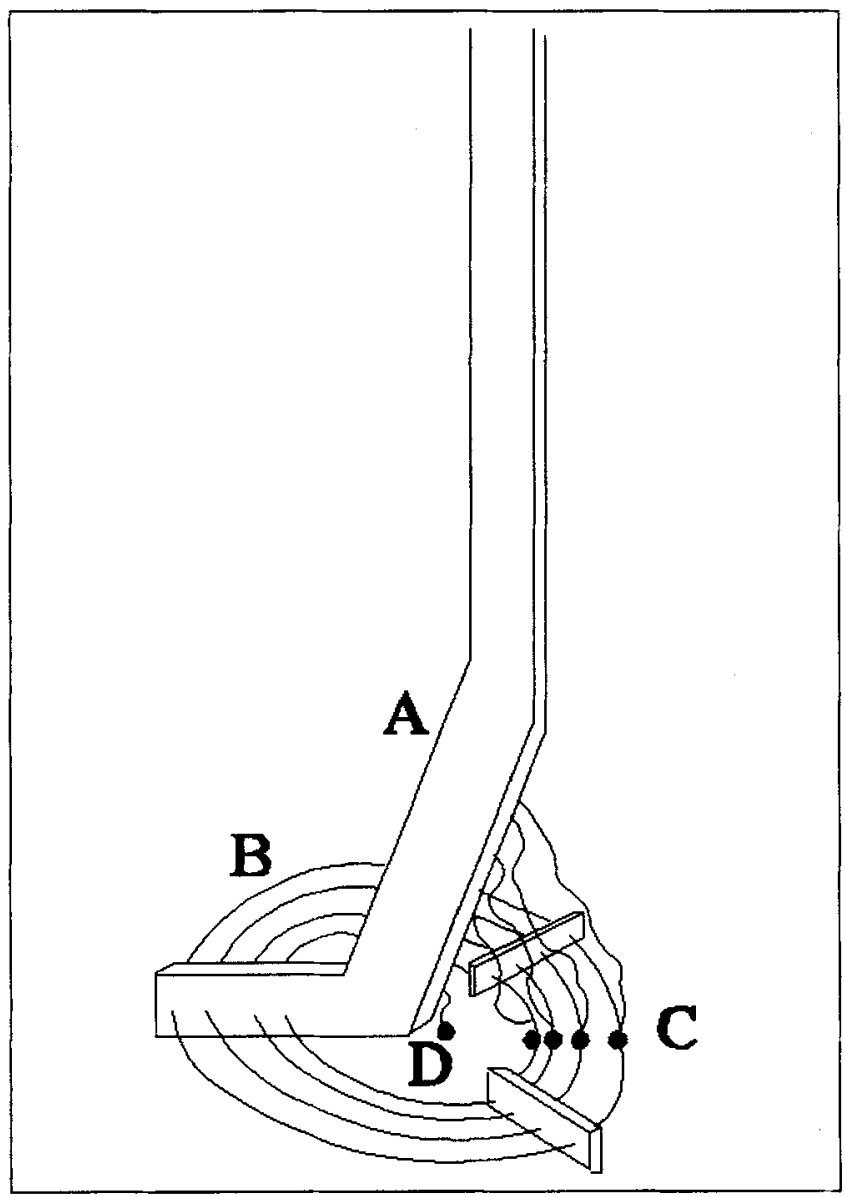

Figure 2. Ring-shaped temperature measurement device developed by Giudici and do Nascimento (1994).

$A$ nylon arm; $B$ four concentric copper rings with a thickness of $2 \mathrm{~mm}$ positioned at $r=8,15,21$, and $27 \mathrm{~mm}$ above a packed bed, with $R_{t}=60 \mathrm{~mm} ; C$ soldered thermocouples; $D$ center line thermocouple. such a device; angular variations in temperature are already filtered by the measurement device itself.

\section{Significance of angular variations}

The three methods just described can be used to even out angular variations and to yield radial profiles suitable for fitting to a two-dimensional continuum deterministic model. Still the question remains whether the variations around the average value can be neglected. To answer this question a parallel can be drawn with the one-dimensional model with a mean cup temperature, Eq. 1 . This model can be used as long as radial temperature gradients are small, otherwise, a two-dimensional model should be chosen (see, e.g., Mears, 1971), who derived a criterion for choosing the model.

The definition of the angular averaged temperature of Eq. 2 is very similar to that of Eq. 1 . The two-dimensional model may be inappropriate if angular variations become too severe. For cases with reaction, the rates evaluated at the average temperature $R[\langle T\rangle]$ will not be equal to the correct average of the angular distributed rates $\langle R[T]\rangle$ in view of the strongly nonlinear dependence of the reaction rate on temperature. Westerink et al. (1992) have proved this for a model in which radial temperature gradients were neglected. As a criterion an error of $5 \%$ is allowed for the ratio of the two rates $f$ :

$$
0.95 \leq \frac{\langle R[T]\rangle}{R[\langle T\rangle]}=f \leq 1.05
$$

Thus knowledge of the amplitude of the angular temperature variation and of the kinetics of the reactions occurring is essential in deciding whether a two-dimensional continuum deterministic model can be used.

\section{Experimental Studies}

\section{Apparatus}

For a detailed discussion of the experimental setup, see Schouten et al. (1994); only the main characteristics of the equipment are cited here. The reactor consists of a vertical tube with a diameter of $53.1 \mathrm{~mm}$ that has been packed with silver on $\alpha$-alumina Raschig rings over a length of $0.506 \mathrm{~m}$. The rings have a diameter of approximately $8.4 \mathrm{~mm}$, a height of about $8.5 \mathrm{~mm}$, and contain a hole with a diameter of about $3.0 \mathrm{~mm}$. The tube is filled with the packing material by dropping the individual particles from the top of the reactor at different angular positions. A random loose packing with a nearly homogeneous distribution of the catalyst is obtained with this "rainy" method (see Šmid et al., 1993), with a porosity of 0.504 . The wall of the reactor is cooled externally with boiling water, the temperature of the water being controlled by pressure variation of the boiling system.

We use the selective oxidation by air of ethene-to-ethene oxide catalyzed by silver on the Raschig rings. This system consists of two reactions occurring in parallel: the epoxidation reaction and the total combustion reaction (see Schouten et al., 1994). We measured the axial concentration profile along the length of the reactor by sampling gas at the wall and analyzing for ethene and carbon dioxide with a gas chromatograph and an infrared meter. 


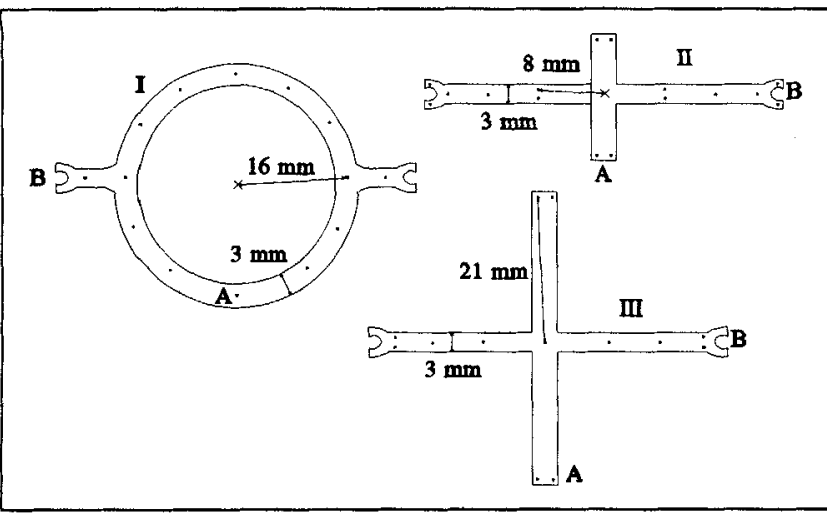

Figure 3. Three different rungs used to fix thermocouples at different angular positions.

I rung at $r=16 \mathrm{~mm}$; II rung at $r=8 \mathrm{~mm}$; IIl rung at $r=21$ $\mathrm{mm}$; A $0.55-\mathrm{mm}$-diameter hole; B special shaped end.

\section{Temperature measurements}

A total of 32 gas temperatures are measured simultaneously in the tube. For this purpose, a ladder-type frame has been placed in the packed bed to keep the thermocouples well fixed at the desired positions. The ladder consists of two hollow rods with a $1.5-\mathrm{mm}$ outside diameter. Between the rods rungs are tightened at different axial positions. The rungs are $2.0 \mathrm{~mm}$ thick and contain several 0.5 -mm-diameter holes. The tip of a K-type thermocouple is introduced from above in such a hole to ensure its exact position. The rung will change the packing arrangement, disturb the flow, and may conduct heat. Schouten et al. (1994) discuss the precautions to minimize these effects.

Three rungs, located at different axial positions, have been used to measure angular variations at 8,16 and $21 \mathrm{~mm}$ distance, respectively, from the centerline of the tube (see Figure 3). Each rung contains eight thermocouples of which the positions are given in Table 1. The other eight thermocouples have been used to measure the radial temperature profile at the inlet and the axial temperature profile in the centerline of the tube.

\section{Method}

The mass flux, wall temperature, and concentration of ethene in the feed have been varied, as given in Table 2. The

Table 1. Angular Position of Thermocouple Defined as the Angle with Respect to Some Arbitrary Reference Point on a Circumference*

\begin{tabular}{cccc}
\hline $\begin{array}{c}\text { Thermocouple } \\
\text { Number }\end{array}$ & \multicolumn{3}{c}{ Distance from Center Line of Tube } \\
\cline { 2 - 4 } & $8 \mathrm{~mm}$ & $16 \mathrm{~mm}$ & $21 \mathrm{~mm}$ \\
\hline 1 & $15^{\circ}$ & $30^{\circ}$ & $11^{\circ}$ \\
2 & $56^{\circ}$ & $60^{\circ}$ & $82^{\circ}$ \\
3 & $112^{\circ}$ & $90^{\circ}$ & $102^{\circ}$ \\
4 & $167^{\circ}$ & $120^{\circ}$ & $170^{\circ}$ \\
5 & $198^{\circ}$ & $180^{\circ}$ & $191^{\circ}$ \\
6 & $232^{\circ}$ & $270^{\circ}$ & $261^{\circ}$ \\
7 & $276^{\circ}$ & $300^{\circ}$ & $286^{\circ}$ \\
8 & $325^{\circ}$ & $330^{\circ}$ & $350^{\circ}$ \\
\hline
\end{tabular}

*Locations of the rungs expressed as distance from the inlet: $5,10,15,25$, 35 and $45 \mathrm{~cm}$
Table 2. Survey of Experimental Operating Conditions

\begin{tabular}{ll}
\hline \multicolumn{1}{c}{ Operating parameter } & \multicolumn{1}{c}{ Conditions } \\
\hline Pressure & $0.5 \mathrm{MPa}$ \\
Wall temperature & $445,465,485 \mathrm{~K}$ \\
Conc. ethene in the feed & $0,0.2,0.5,0.9,1.2,1.7,2.0 \mathrm{~mol} \%$ \\
Mass fluxes & $0.41,0.82,1.64 \mathrm{~kg} /\left(\mathrm{m}^{2} \cdot \mathrm{s}\right)$ \\
Superficial velocity & $0.11-0.46 \mathrm{~m} / \mathrm{s}$ \\
\hline
\end{tabular}

wall temperature and ethene concentration have a direct influence on the reaction rates. At reaction conditions, the temperature of the feed is equal to that of the wall so that the temperature is constant over the inlet plane.

In heat transfer experiments nitrogen is used as gas. We varied the wall temperature and mass flux; the preheat temperature was set in such a way that the temperature in the center of the inlet plane of the tube was about $40^{\circ} \mathrm{C}$ lower than the wall temperature, so in the heat transfer experiments the inlet temperature profile was not flat.

Angular temperature variations have been measured at six axial positions in the tube. We had to open the reactor five times and change the position of the rungs in the frame because the temperature measurement device allows for measurement of angular variations at a certain radial position for one axial position only. The tube was filled again with the same catalyst material after each modification of the thermocouple frame. Packing heights were observed to be the same after repacking, if the same method of filling was applied. After repacking, the tube was operated under reaction conditions for a certain period of time-the conversion was observed to increase during the first hours of reaction-under standard conditions until a stable activity was reached. The total conversion remained equal within $10 \%$ under standard conditions for the six packings. We once repacked the bed and maintained the same thermocouple configuration to study the effect of only repacking.

\section{Results}

\section{Angular temperature variations}

An example of the variation in temperature as measured around a radial circumference is given in Figure 4 as the difference with the mean temperature at a certain angular position. The smooth lines drawn through the eight experimental points have been constructed with a spline technique and do not represent the true course of the angular temperature variations. In this particular experiment the angular temperature deviations are maximally $6 \mathrm{~K}$. This deviation is significant with respect to the total temperature difference over the radius of about $15 \mathrm{~K}$. The possible deviation of $0.5 \mathrm{~mm}$ in the thermocouple location will result in a possible temperature deviation of only $0.6 \mathrm{~K}$ for the experiment of Figure 4. Therefore, the observed angular variation cannot be a result of the uncertainty in the location of the temperature sensor.

By inspection of the angular temperature profiles we determine whether there is some periodicity in angular temperature variations caused by a (semi-) ordered packing arrangement. If any periodicity is present, it probably will be related to the particle diameter. Therefore the length one particle occupies on a circumference is shown in Figure 4 for a radius of $21 \mathrm{~mm}$ from the tube centerline. In the profile of Figure 4, 


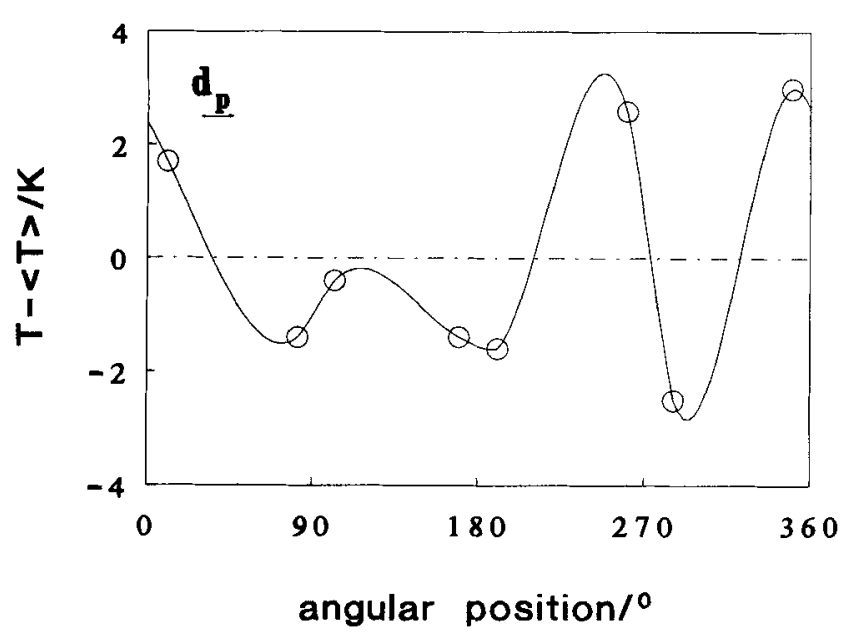

Figure 4. Temperature variations around the mean temperature as measured at 8 different angular locations.

Conditions are $C_{E}=1.7 \mathrm{~mol} \%, T_{w}=466 \mathrm{~K}, \phi_{m}=1.62$ $\mathrm{kg} / \mathrm{m}^{2} \mathrm{~s}, z=0.25 \mathrm{~m}, r=21 \mathrm{~mm}$

and also in all other angular profiles, no regular periodical oscillation in temperature could be detected, (see also Dixon, 1993). Therefore, the variations are considered to be random.

Because the angular temperature variations are random, they can be characterized by the mean temperature of Eq. 2 and a standard deviation $\sigma$, which describes the variation around the mean temperature:

$$
\sigma / K=\sqrt{\sum_{i=1}^{N} \frac{\left(T_{i}-\langle T\rangle\right)^{2}}{N-1}} / K
$$

in which $N$ is the number of different angular measurements.

The accuracy of the thermocouples has been tested for an isothermal bed with the inlet temperature equal to the wall

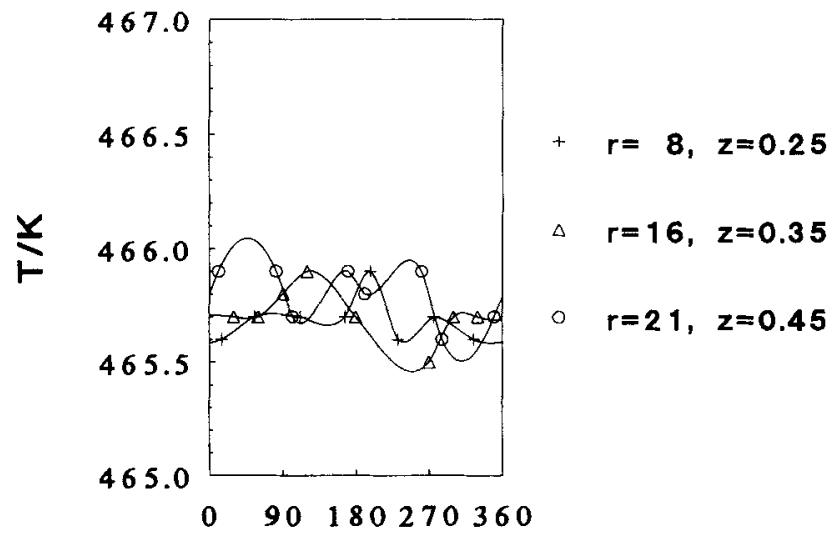

angular position $/^{\circ}$

Figure 5. Temperature variations for a packed bed with a uniform temperature being equal to the wall temperature.

Conditions are $T_{w}=465.6 \mathrm{~K}$ and $\phi_{m}=0.81 \mathrm{~kg} / \mathrm{m}^{2}$.

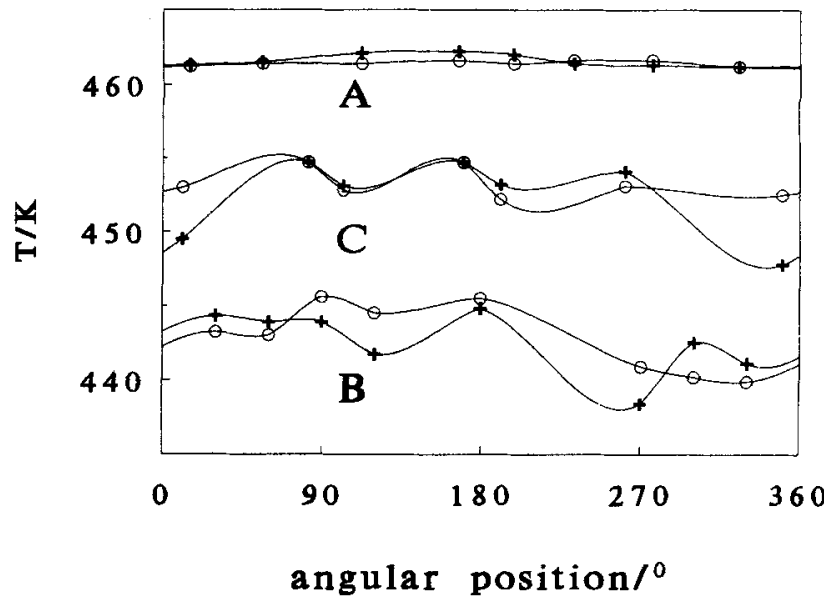

Figure 6. Comparison of temperature variations in two packed beds with reaction.

Conditions are $T_{w}=464.6 \mathrm{~K}, \phi_{m}=0.81 \mathrm{~kg} / \mathrm{m}^{2} \mathrm{~s}$. Position A is $z=0.05 \mathrm{~m}$ and $r=16 \mathrm{~mm}$, near the inlet, position $B$ is $z=0.10 \mathrm{~m}$ and $r=21 \mathrm{~mm}$, and position $C$ is $z=0.35 \mathrm{~m}$ and $r=8 \mathrm{~mm}$, which is beyond the hot spot.

temperature, so a uniform temperature is expected in the tube in case the different thermocouples are accurate. An angular temperature profile of such an experiment is shown in Figure 5: the temperatures are equal within $0.3 \mathrm{~K}$.

In the following the influence of different parameters on the angular temperature variations is discussed by considering possible trends in the standard deviations. An explanation for the observed trends is given in the discussion.

\section{Influence of repacking}

The effect of repacking the bed while maintaining the same thermocouple configuration is shown in Figure 6 for a case with reaction. Angular temperature profiles at three positions in the repacked bed are compared with those of the original bed at the same operating conditions. A different distribution of angular variations is found for the different packing arrangements obtained. The angularly averaged temperature and the standard deviations in the two beds are compared for the three positions (see Table 3). Also the total conversions over the two beds are compared. The angularly averaged temperature of the two packed beds are almost equal for all three positions, and also the conversions are equal. On the other hand, the standard deviations of the two beds differ for two of the three positions, $\sigma$ being about twice as large for the second bed for positions $A$ and $C$. Thus the standard deviation at a certain axial position and on the radial circumference at the same operating conditions is not unique but different from packing to packing.

Table 3. Angularly Averaged Temperatures and Standard Deviations in Two Packed Beds at Equal Operating Conditions and for Three Different Positions*

\begin{tabular}{cccccccc}
\hline Bed & $\zeta$ & $\begin{array}{c}\langle T\rangle_{A} \\
\mathbf{K}\end{array}$ & $\begin{array}{c}\langle T\rangle_{B} \\
\mathbf{K}\end{array}$ & $\begin{array}{c}\langle T\rangle_{C} \\
\mathbf{K}\end{array}$ & $\begin{array}{c}\sigma_{A} \\
\mathbf{K}\end{array}$ & $\begin{array}{c}\sigma_{B} \\
\mathbf{K}\end{array}$ & $\begin{array}{c}\sigma_{C} \\
\mathbf{K}\end{array}$ \\
\hline 1 & 0.405 & 461.4 & 442.6 & 453.3 & 0.17 & 2.32 & 1.01 \\
2 & 0.407 & 461.6 & 442.9 & 452.4 & 0.41 & 2.30 & 2.71 \\
\hline
\end{tabular}

*See also Figure 6. 


\section{Influence of mass flux}

The bed has not been repacked for the experiments at different mass fluxes. Hence, the distribution of the variations is the same (see Figures $7 a$ and $8 a$ ). Both under reaction conditions as well as without reaction, the amplitude changes for a change in mass flux. In case of heat-transfer experiments, the standard deviations $\sigma$ of the angular temperature variations are about the same near the inlet for different mass fluxes. However, they increase at increasing mass fluxes at positions further downstream in the bed (see Figure $7 b$ ). When a reaction is executed (see Figure 8b), $\sigma$ decreases near the inlet for an increasing mass flux. A maximum in $\sigma$ is found at positions further downstream in the bed: these maxima coincide with the hot spot.

\section{Influence of position}

To evaluate the effect of axial and radial position on angular temperature variations, results from different repackings

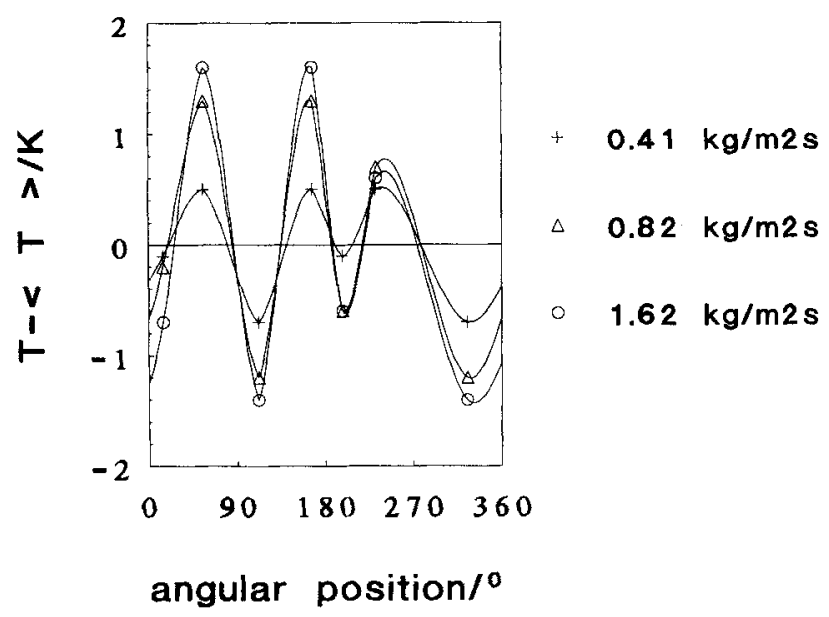

(a)

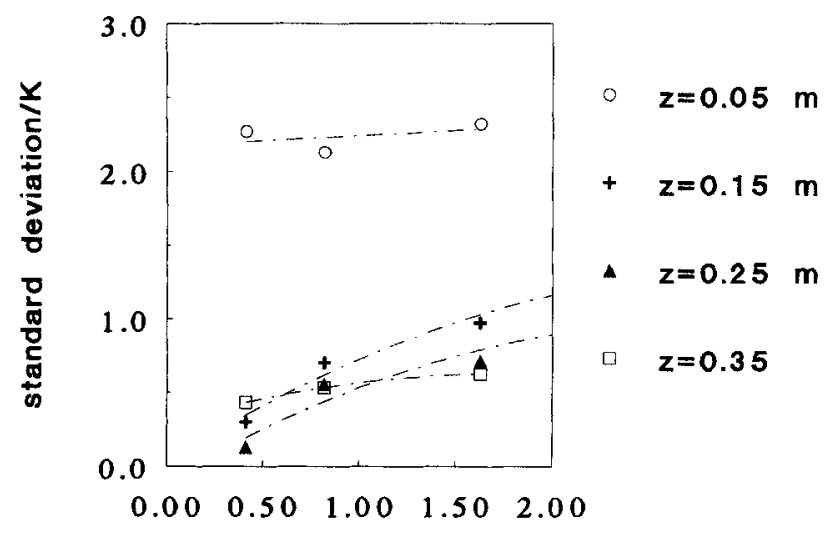

mass flux $/ \mathrm{kg} / \mathrm{m}^{2} \mathrm{~s}$

(b)

Figure 7. Comparison of (a) temperature variations and (b) standard deviations for heat transfer experiments in a packed bed and for different mass fluxes.

Conditions are $T_{w}=465.1$, and (a) $z=0.25 \mathrm{~m}$ and $r=21 \mathrm{~mm}$ or (b) $r=8 \mathrm{~mm}$ and different axial locations.

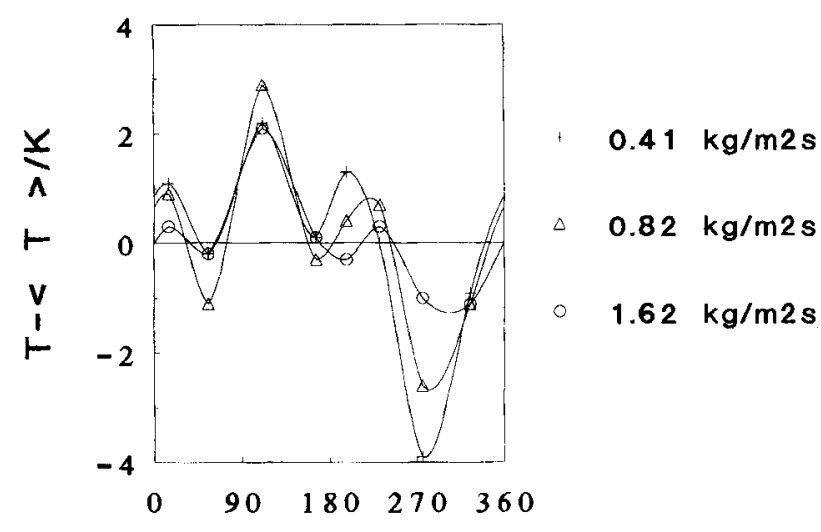

angular position $/^{\circ}$

(a)

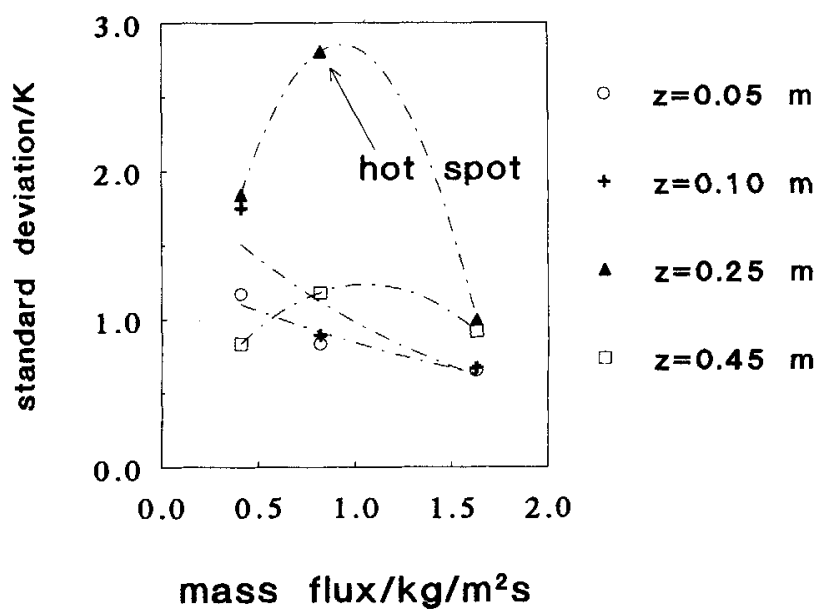

(b)

Figure 8. Comparison of (a) temperature variations and (b) standard deviations for experiments with reaction in a packed bed and for different mass fluxes.

Also the hot-spot location is shown for one of the mass fluxes. Conditions are $T_{w}=465.7, C_{E}=0.9 \mathrm{~mol} \%, z=0.25$ $\mathrm{m}$, and $r=16 \mathrm{~mm}$.

have to be compared. Such a comparison may be disguised, because each repacking itself already leads to different angular temperature distributions and different standard deviations. Therefore only some general trends can be distinguished.

The dependence of $\sigma$ on the axial location is shown in Figure 9. In heat-transfer experiments the general trend is a decrease in $\sigma$ the further away from the inlet it is. In an experiment with reaction a maximum in $\sigma$ is found, its location coinciding with the location of the hot spot.

In Figure 10 the dependence of $\sigma$ on the radial position is shown for both heat transfer as well as reaction conditions. The scatter in the data points is large and no trend can be distinguished.

\section{Influence of reaction}

Experiments with and without reaction have already been compared in the two former sections. The temperature level 


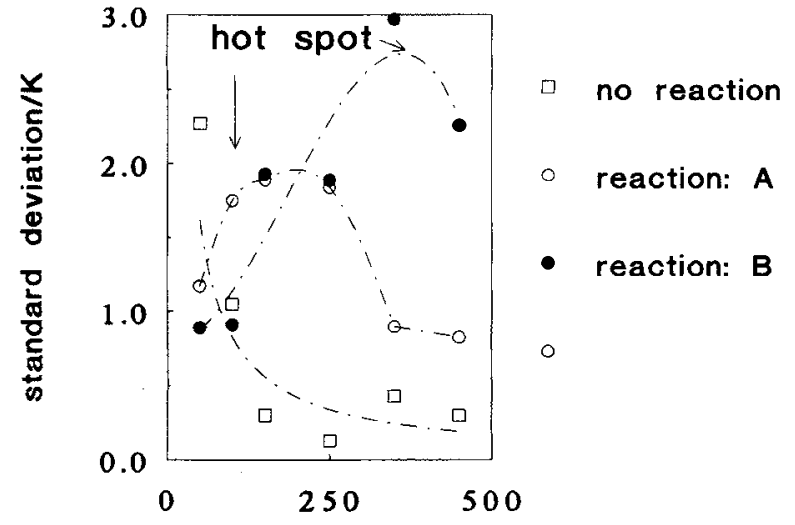

axial position/m

Figure 9. Standard deviation of angular temperature variations as a function of the axial position in the packed bed.

Also the hot-spot locations are shown. Conditions without reaction are $\phi_{m}=0.41 \mathrm{~kg} / \mathrm{m}^{2} \mathrm{~s}, T_{w}=465 \mathrm{~K}$, and $r=16 \mathrm{~mm}$, and with reaction $T_{w}=465 \mathrm{~K}$, and for case $\mathrm{A}: \phi_{m}=0.41$ $\mathrm{kg} / \mathrm{m}^{2} \cdot \mathrm{s}, r=16 \mathrm{~mm}$, and $C_{E}=0.9 \mathrm{~mol} \%$, and case $\mathrm{B}: \phi_{m}=$ $1.61 \mathrm{~kg} / \mathrm{m}^{2} \cdot \mathrm{s}, r=16 \mathrm{~mm}$, and $C_{E}=1.7 \mathrm{~mol} \%$.

through a change in the wall temperature does not itself influence the amplitude of angular variations. Almost the same amplitude of angular variations is found for the same packing (see Table 4). Experimental results for angular temperature variations as a function of the ethene feed concentration are shown in Figure 11 and of the wall temperature in Figure 12, respectively. Angular variations become larger if ethene concentration and/or wall temperature increase; that is when the reaction rates become faster.

\section{Discussion}

The results seem to indicate that angular temperature variations are related to the radial temperature difference. In Figure 13a the standard deviation is plotted vs. the total radial temperature difference $T_{\text {center }}-T_{w}$, and in Figure 13b it is plotted vs. the radial temperature gradient $d T / d r$. This local gradient was calculated with a second-order polynomical equation that was determined from the radial measurements, taking radial symmetry into account. Data for the heat-transfer experiments are distinguishable from data from reaction experiments because the temperature difference is negative, so that they are on the left side of the $y$-axis in Figure 13a. In both graphs an almost linear dependence of $\sigma$ is observed. Therefore we correlated the standard deviation linearly to the total radial temperature difference and found the following relation to fit the data with an average error of $30 \%$ :

$$
\sigma=0.2+0.05 *\left|\left(T_{\text {center }}-T_{w}\right)\right| \text {. }
$$

No statistically significant dependence of $\sigma$ on any other parameter could be detected in the residuals $\sigma_{\text {exp }}-\sigma_{\text {calc }}$, where $\sigma_{\text {calc }}$ is determined on the basis of Eq. 5. This is shown in Figure 14 for the radial temperature gradient, the mass flux, the feed concentration of ethene, and the wall temperature, respectively: all data points are randomly distributed

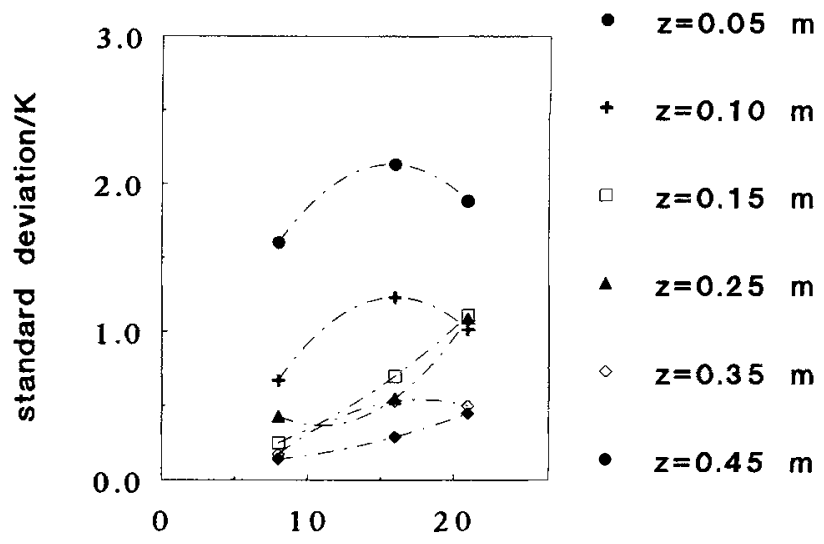

radial position $/ \mathrm{mm}$

(a)

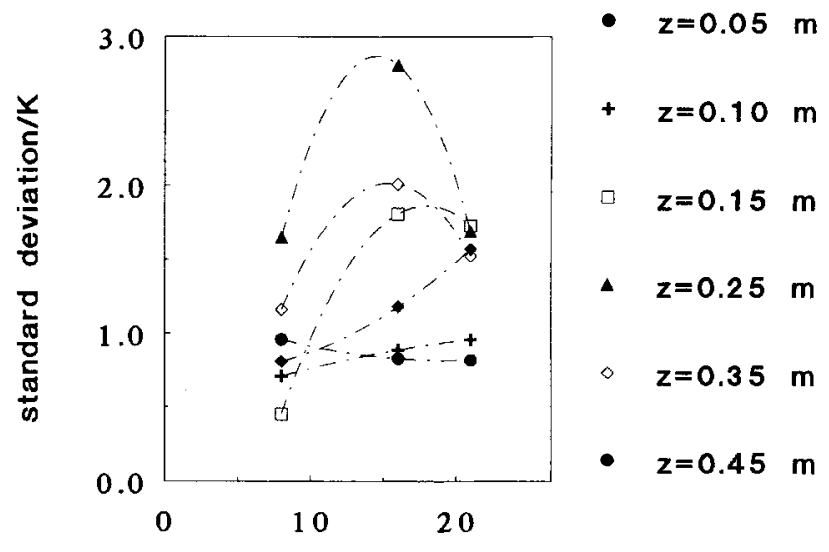

radial position $/ \mathrm{mm}$

(b)

Figure 10. Standard deviations of angular temperature variations as a function of the radial position.

Conditions in (a) are without reaction $\phi_{m}=0.81 \mathrm{~kg} / \mathrm{m}^{2} \cdot \mathrm{s}$ and $T_{w}=465 \mathrm{~K}$. In (b) with reaction we have $T_{w}=465 \mathrm{~K}$ $\phi_{m}=0.81 \mathrm{~kg} / \mathrm{m}^{2} \cdot \mathrm{s}$ and $C_{E}=0.9 \mathrm{~mol} \%$, and the hot spot is located at $z=0.25 \mathrm{~m}$.

around the horizontal axis, so these parameters do not influence $\sigma$. The large scatter around the horizontal axis may partly be explained by the fact that several repackings are compared.

Table 4. Standard Deviations of Angular Temperature Variations for Different Wall Temperatures*

\begin{tabular}{ccccc}
\hline$\sigma(\mathrm{K})$ & $\mathrm{T}(\mathrm{K})$ & 445 & 465 & 485 \\
\hline (I) & & & & \\
(II) & & 1.07 & 1.11 & 1.00 \\
(III) & 0.43 & 0.43 & 0.51 \\
\hline
\end{tabular}

*Conditions are (I) $\phi_{m}=0.81 \mathrm{~kg} / \mathrm{m}^{2} * \mathrm{~s}, r=21 \mathrm{~mm}$, and $z=0.15$; (II) $\phi_{m}$ $=0.41 \mathrm{~kg} / \mathrm{m}^{2} \cdot \mathrm{s}, r=16 \mathrm{~mm}$, and $z=0.35$; (III) $\phi_{m}=1.62 \mathrm{~kg} / \mathrm{m}^{2} \cdot \mathrm{s}, r=8$ $\mathrm{mm}$, and $z=0.05$. 


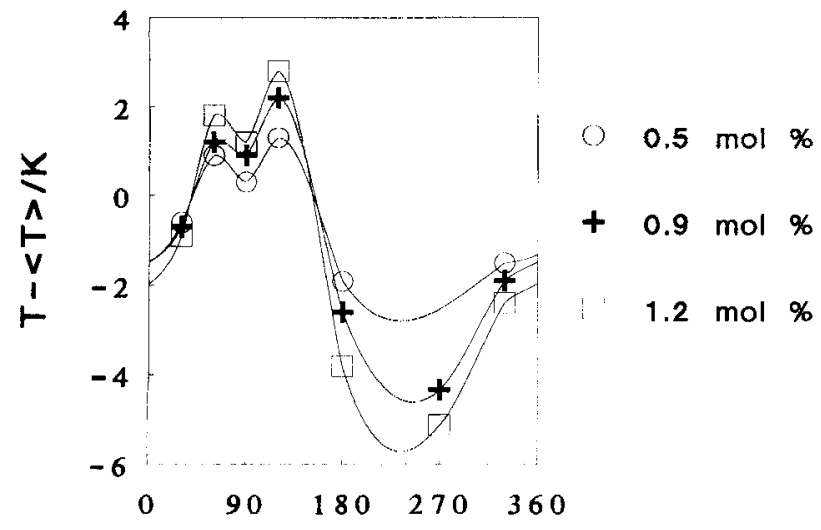

angular position ${ }^{\circ}$

Figure 11. Angular temperature variations as a function of the ethene concentration in the feed.

Conditions are $T_{w}=465 \mathrm{~K}, \phi_{m}=0.81 \mathrm{~kg} / \mathrm{m}^{2} \cdot \mathrm{s}, z=0.15 \mathrm{~m}$, and $r=16 \mathrm{~mm}$.

The apparent influence of the different parameters is observed on the amplitude of angular temperature variations because the axial and radial temperature profiles also change. The observed trends are:

- In the heat-transfer experiments $\sigma$ decreases over the length of the reactor because the radial temperature differences become smaller further downstream in the bed.

- $\sigma$ increases at increasing ethene concentrations/wall temperatures because reaction rates become higher, and thus radial temperature differences larger.

- In the heat-transfer experiments $\sigma$ is constant at the inlet because the radial temperature difference has deliberately been set to the constant value of $40^{\circ} \mathrm{C}$ in each experiment.

- In the heat-transfer experiments $\sigma$ increases if the mass flux increases, because local radial temperature differences near the bed exit are larger at higher mass fluxes, because the gas needs a longer distance to cool down.

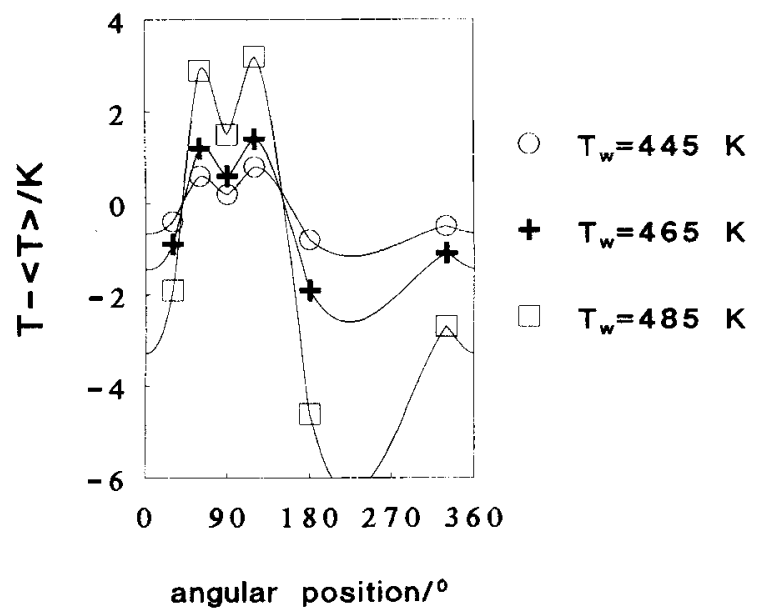

Figure 12. Angular temperature variations as a function of wall temperature.

Conditions are $\phi_{m}=1.85 \mathrm{~kg} / \mathrm{m}^{2} \cdot \mathrm{s}, z=0.15 \mathrm{~m}$, and $r=16$ mm.

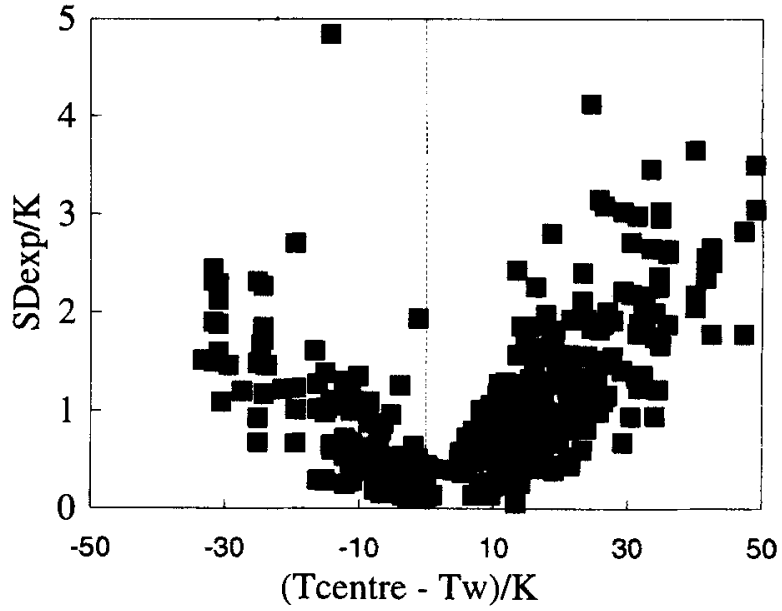

(a)

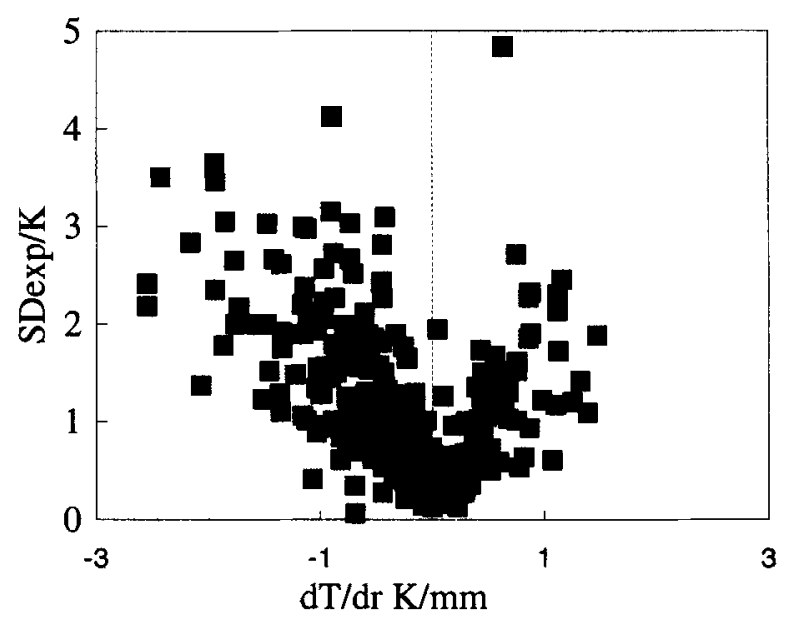

(b)

Figure 13. Standard deviation of angular temperature variations as a function of (a) the total radial temperature difference and (b) the local radial temperature gradient.

- In experiments with reaction, $\sigma$ first increases in the axial direction until it reaches a maximum in the hot spot where the total radial temperature difference is largest; after that it decreases further downstream in the bed. Changing the mass flux moves the hot spot (see Schouten et al., 1994): whether $\sigma$ will increase or decrease depends on the location under consideration compared to the hot-spot location.

We still must determine whether a continuum model can be applied. Thus, we apply Eq. 3 and use a probability density function to characterize the distribution of temperatures. The temperatures are distributed normally around the angularly averaged temperature, if the angular variations are truly random. For the probability density function $p\left[T_{i}\right]$ we can write

$$
p\left[T_{i}\right]=\frac{1}{\sigma \sqrt{2 \pi}} e^{-\left(T_{i}-\langle T\rangle\right)^{2} / 2 \sigma^{2}}
$$




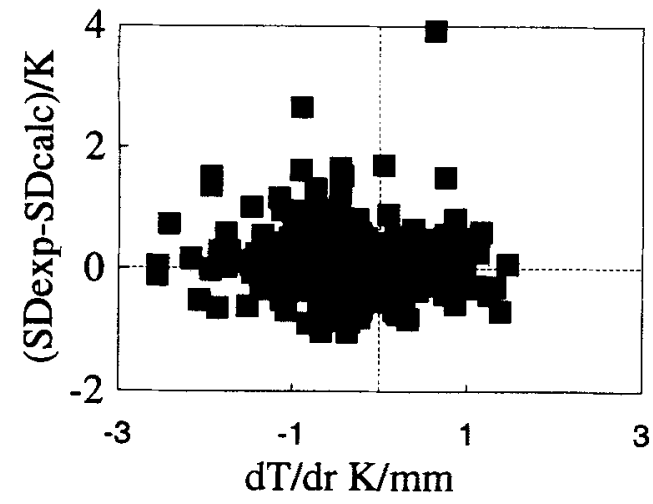

(a)

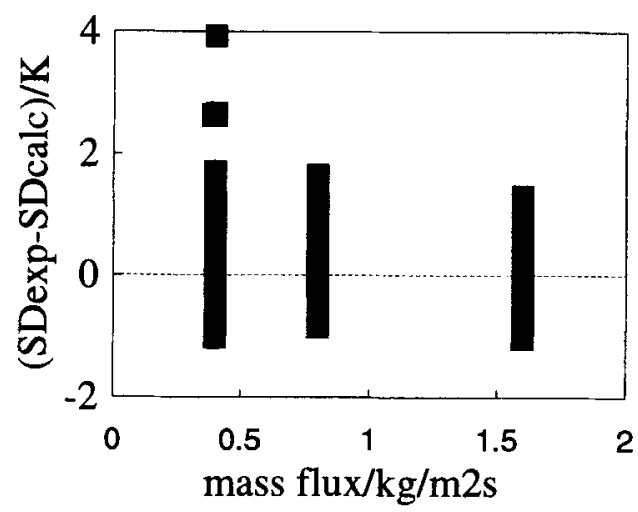

(b)

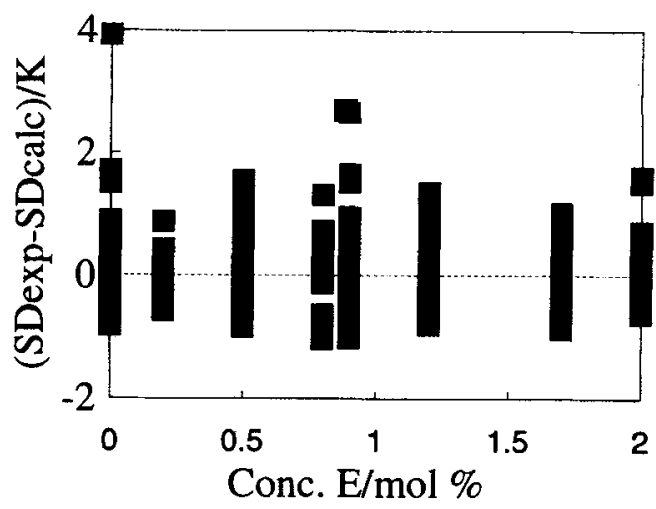

(c)

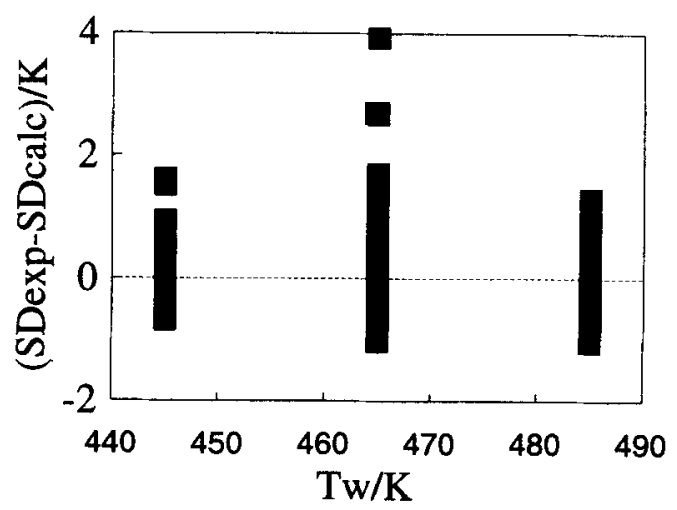

(d)

Figure 14. Residuals of the standard deviations of angular temperature variations.

(a) Radial temperature gradient, (b) mass flux, (c) ethene concentration in the feed, and (d) wall temperature.

The mean reaction rate over a circular circumference $\langle R(T)\rangle$ in Eq. 3 can be expressed as follows:

$$
\langle R(T)\rangle=\frac{\int_{T=-\infty}^{\infty} R(T) \cdot p(T) \cdot d T}{\int_{T=-\infty}^{\infty} p(T) \cdot d T}
$$

The kinetics of the ethene epoxidation as reported by Schouten et al. (1994) (see Table 5) have been used to calculate the reaction rates at five different conditions for which a relatively large angular temperature variation was observed. The ratios between the rates $f_{i}$ have been calculated for these

Table 5. Reaction Rate Expressions for Epoxidation (1) and Total Combustion Reaction (2)

$$
\begin{aligned}
& \text { Epoxidation reaction: } \\
& R_{1}=\frac{1.20 \times 10^{4} \cdot e^{-5,790 / r} P_{E} P_{O}}{\left(1+0.022 \cdot e^{3,000 / T} P_{E}+1.5 P_{O}+100 P_{E O}+60 P_{C}+40 P_{H}\right)^{2}}
\end{aligned}
$$

Total combustion reaction

$$
R_{2}=\frac{6.06 * 10^{7} \cdot e^{-9,200 / T} P_{E} P_{O}}{\left(1+0.0065 \cdot e^{3,600 / T} P_{E}+2.95 P_{O}+250 P_{E O}+130 P_{C}+55 P_{H}\right)^{2}}
$$

five conditions and are given in Table 6. A larger difference from one is found for the total combustion reaction compared for the epoxidation reaction because the greater apparent activation energy shows that the reaction rates depend more on the temperature. All values for $f_{i}$ are close to one, so Eq. 3 is satisfied. Hence, the angular variations have only a limited effect on the calculated reaction rates.

The angular variations have a greater impact on the reaction rates whenever temperature differences are larger and/or reactions with larger activation energies occur. In addition, different results may be obtained if different particle shapes are used, the method of packing is changed, or the number of particles on a tube diameter is different.

Table 6. Ratio $f_{i}$ of the Angularly Averaged Reaction Rate to the Rate Determined at the Angularly Averaged Temperature for Epoxidation (1) and Total Combustion Reaction (2) for an Arbitrary Chosen Number of Cases

\begin{tabular}{lcccccc}
\hline Case & $\begin{array}{c}z \\
(\mathrm{~m})\end{array}$ & $\begin{array}{l}r \\
(\mathrm{~mm})\end{array}$ & $\begin{array}{l}\langle T\rangle \\
(\mathbf{K})\end{array}$ & $\begin{array}{c}\sigma \\
(\mathbf{K})\end{array}$ & $f_{1}$ & $f_{2}$ \\
\hline A & 0.25 & 21 & 458.7 & 3.46 & 1.006 & 1.013 \\
B & 0.25 & 21 & 480.3 & 4.12 & 1.005 & 1.013 \\
C & 0.05 & 8 & 475.2 & 1.29 & 1.001 & 1.001 \\
D & 0.15 & 16 & 479.0 & 1.93 & 1.001 & 1.003 \\
E & 0.35 & 16 & 519.2 & 3.05 & 1.002 & 1.005 \\
\hline
\end{tabular}




\section{Conclusions}

Temperatures at different angular positions have been observed to differ up to $10 \mathrm{~K}$ in some cases, making it necessary to average the angularly measured temperatures to obtain "correct" temperatures. No periodicity has been found in the variations, so their amplitude can be characterized with a standard deviation.

Repacking the bed leads to the same mean temperatures and bed conversions despite the differences in the standard deviations found after repacking.

We found angular temperature variations to be dependent on the total radial temperature difference. Therefore the largest angular variations have been found near the inlet in heat-transfer experiments and near the hot spot in reaction experiments. Other parameters considered, such as the mass flux, the wall temperature, the concentration ethene in the feed, and the position in the tube, only have an indirect impact on the amplitude of the angular temperature variations in the way in which they change the radial temperature profiles.

Application of the criterion of Eq. 3 shows that angular variations have only a small effect on the reaction rates in our case. Although the statistical character of the packed bed results in significant temperature variations, we can neglect these if an angular averaged temperature is used. Thus, a two-dimensional deterministic continuum model can be applied in our case.

\section{Acknowledgments}

The authors would like to thank J. G. H. Borkink and P. C. Borman for the discussions, M. J. A. M. Elshout for the experimental work, and G. Banis, A. H. Pleiter, J. Rooker, and O. D. Veehof for the technical support.

\section{Notation}

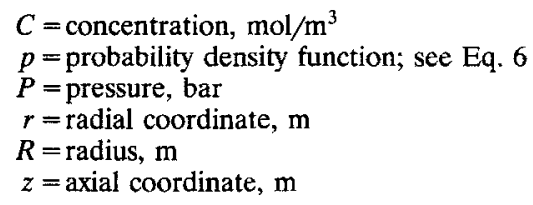

\section{Greek letters}

$$
\begin{aligned}
\theta & =\text { angle } \\
\zeta & =\text { conversion } \\
\phi_{m} & =\text { mass flux }, \mathrm{kg} /\left(\mathrm{m}^{2} \cdot \mathrm{s}\right)
\end{aligned}
$$

\section{Subscripts and superscripts}

$$
\begin{aligned}
C & =\text { carbon dioxide } \\
\text { calc } & =\text { calculated } \\
\mathrm{E} & =\text { ethene } \\
\mathrm{EO} & =\text { ethene oxide } \\
\exp & =\text { experimental }
\end{aligned}
$$

$$
\begin{aligned}
H & =\text { water } \\
O & =\text { oxygen } \\
t & =\text { tube } \\
w & =\text { wall } \\
1 & =\text { of the epoxidation reaction } \\
2 & =\text { of the total combustion reaction }
\end{aligned}
$$

\section{Literature Cited}

Borkink, J. G. H., C. G. Watering, and K. R. Westerterp, "The Statistical Character of Bed-Scale Effective Heat Transport Coefficients for Packed Beds," Trans. Ind. Chem. Eng., 70A, 610 (1992).

Borkink, J. G. H., and K. R. Westerterp, "Significance of the Radial Porosity Profile for the Description of Heat Transport in WallCooled Packed Beds," Chem. Eng. Sci., 49, 863 (1994).

Borman, P. C., and K. R. Westerterp, "An Experimental Study of the Selective Oxidation of Ethene in a Wall Cooled Tubular Packed Bed Reactor," Chem. Eng. Sci., 47, 2541 (1992).

Cresswell, D. L., "Heat Transfer in Packed Bed Reactors," Chemical Reactor Design and Technology, H. I. de Lasa, ed., Nijhoff, Dordrecht, The Netherlands, p. 687 (1986).

Dixon, A. G., and W. R. Paterson, "Heat Transfer in Packed Beds of Low Tube/Particle Diameter Ratio," ACS Symp. Ser., 20, 238 (1978).

Dixon, A. G., "Wall and Particle-Shape Effects on Heat Transfer in Packed Beds," Chem. Eng. Commun., 71, 217 (1988).

Dixon, A. G., "Angular Temperature Variations in Fixed Beds of Spheres," Proc. Nat. Heat Transfer Conf., Atlanta, p. 55 (1993).

Dixon, A. G., "Heat Transfer in Packed Beds of Spheres with $d t / d p$ < 4," Proc. Int. Heat Transfer Conf., Brighton, UK, Inst. Chem. Engrs. Symp. Ser., 135, 225 (1994).

Fedoseev, V. N., and O. I. Shanin, "Statistical Characteristics of Convective Exchange in a Stationary Granular Bed," Inz.-Fiz. $Z h$, 51, 194 (1986).

Giudici, R., and do Nascimento, C. A. O., "Analysis of a Ring-Shaped Sensor for Use in Packed-Bed Heat Transfer Studies," Can. J. Chem. Eng., 72, 43 (1994).

Hoffman, H., "Progress in Modelling of Catalytic Fixed-Bed Reactors," Ger. Chem. Eng., 2, 258 (1979).

Mears, D. E., "Diagnostic Criteria for Heat Transport Limitations in Fixed Bed Reactors," J. Catal., 20, 127 (1971).

Schouten, E. P. S., P. C. Borman, and K. R. Westerterp, “Oxidation of Ethene in a Wall-Cooled Packed-Bed Reactor," Chem. Eng. Sci., 24b, 4725 (1994).

Šmid, J., P. V. Xuan, and J. Thýn, "Effect of Filling Method on the Packing Distribution of a Catalyst Bed," Chem. Eng. Tech., 16, 114 (1993).

Vortmeyer, D., and E. Haidegger, "Discrimination of Three Approaches to Evaluate Heat Fluxes for Wall-Cooled Fixed Bed Chemical Reactors," Chem. Eng. Sci., 46, 2651 (1991).

Westerink, E. J., N. Koster, and K. R. Westerterp, "The Choice between Cooled Tubular Reactor Models; Analysis of the Hot Spot," Chem. Eng. Sci., 45, 3443 (1990).

Wijngaarden, R. J., and K. R. Westerterp, "Incorporation of Statistical Distributions of Particle Properties in Chemical Reactor Design and Operation: The Cooled Tubular Reactor," Chem. Eng. Sci., 47, 1881 (1992a).

Wijngaarden, R. J., and K. R. Westerterp, "The Statistical Character of Packed-Bed Heat Transport Properties," Chem. Eng. Sci., 47, 3125 (1992b).

Manuscript received Oct. 13, 1995, and revision received Feb. 5, 1996 